\title{
Algunas reflexiones sobre "Logística y desarrollo territorial" (RTT 17/2017)
}

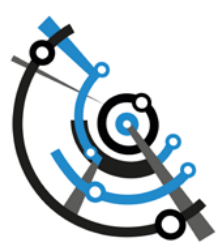

\author{
Gian Paolo Torricelli \\ Osservatorio dello Sviluppo Territoriale, Accademia di Architettura, Università della Svizzera \\ Italiana - Mendrisio, Suiza.
}

\begin{abstract}
El Dossier "Logística y desarrollo territorial” (RTT 17 / 2017) ofrece una visión general de un sector en rápido crecimiento, no solo como sistema de transporte, sino como soporte estratégico para el avance industrial y del comercio mayorista (y minorista en línea), en la perspectiva de un desarrollo territorial sostenible. Los artículos proponen interesantes reflexiones teóricas y casos de estudio -en particular sobre el desarrollo reciente de la "logística urbana" en distintos contextos (Palacios-Argüello et al. 2017; Muñuzuri et al. 2017; Pachón Rincón et al. 2017)- así como una investigación del impacto de la certificación Fair Trade (comercio justo) en una red de cooperativas apícolas en el nordeste brasileño (Rosado de Sousa et al. 2017) y un análisis de los flujos ferroviarios de carga generados por la industria automotriz mexicana (García-Ortega et al. 2017).
\end{abstract}

El dossier parece estar bien construido, especialmente para lo que concierne, entre otras cosas, el juego entre los actores involucrados (públicos y privados), la arquitectura de los flujos logísticos y los principales problemas encontrados en grandes áreas urbanas. Sin embargo, la discusión sobre el desarrollo territorial podría haberse investigado más desde el punto de vista de los impactos propiamente espaciales de las actividades logísticas. En la presentación, los autores afirman oportunamente que “...el diseño, análisis y desarrollo logístico está vinculado al desarrollo territorial. Desde un enfoque de sistemas, la proyección territorial de las actividades logísticas es un complemento fundamental que permite completar los ángulos de análisis necesarios para una comprensión integral de la actividad humana..." (González-Feliu y Cedillo-Campos 2017:1). Así, "la logística ha tomado un rol principal tanto a nivel local, como nacional e internacional". La fluidez logística es hoy una meta que no sería posible alcanzar sin considerar el componente territorial en donde las organizaciones desarrollan sus actividades" (Ibíd.).

En el dossier se reconocen, también, los impactos negativos del crecimiento reciente de estas actividades, que por un lado son un vector de desarrollo económico y de creación de valor y, por otro lado, producen externalidades negativas (congestión, ruido, contaminación del aire, etc.) que se expresan cada vez más en las áreas urbanas. Tratándose de "logística y desarrollo territorial", sin embargo, me pregunto si los conflictos pueden ser limitados al tráfico, a los horarios (temporalidades), a las dificultades del suministro o a la competencia entre empresas (Muñuzuri et al. 2017:44-45). El monitoreo y la contención de estas externalidades son fundamentales para la calidad de vida de una ciudad. Sin embargo, hay otros impactos directamente relacionados al 
asentamiento de la logística que no pueden ser ignorados. Primero: con el desarrollo cada vez más importante de las actividades logísticas en la organización misma de las empresas, tenemos que esperar el crecimiento de problemas resultantes del consumo de tierra o, mejor dicho, del consumo de suelo urbano en las zonas industriales suburbanas y también en los centros de abastecimiento de espacios metropolitanos (hub locales, terminales de carga y depósitos especializados). Segundo: no aparece una reflexión explícita sobre la lógica del asentamiento (de la territorialización) de las empresas logísticas (ver Raimbault et al. 2013). De hecho, en el contexto de un proyecto de investigación, hemos comprobado que esas empresas generalmente no tienen la misma relación con el territorio que lo está hospedando, en comparación con las otras actividades de producción y servicio y ubicadas en los mismos lugares (generalmente en zonas industriales suburbanas).

\section{El impacto espacial y el consumo de tierra de la logística}

La logística es un sector empresarial en constante crecimiento, desencadenado principalmente por la liberalización de los intercambios económicos y las innovaciones tecnológicas. Hoy en día se beneficia de la globalización, no solo en términos de transporte de bienes, sino que también es capaz de desarrollar un marco integrado de suministro de bienes (comúnmente definido como Supply Chain Management) que incluye una variedad de servicios adicionales. Las actividades logísticas abarcan, por lo tanto, una configuración de servicios cada vez más especializados, directamente al soporte de las empresas, como la planificación del despacho en función de los ritmos de producción, el almacenamiento, el embalaje y el etiquetado, el seguimiento y los inventarios de productos, hasta la custodia de valores en zonas extraterritoriales (puertos francos).

Cómo bien afirma el Dossier, la logística opera para optimizar los flujos de bienes y respalda otros segmentos económicos como (entre otros) los fabricantes tradicionales y avanzados, mayoristas y grandes minoristas o la industria alimentaria (ver el ejemplo del food $h u b$ ), que facilita la producción de valores adicionales agregados a sus productos, por ejemplo, racionalizando los despachos personalizados o el proceso de marca basado en el lugar (competencia del made in o hecho en). Estos servicios son la esencia del modelo económico y el núcleo de la competitividad entre las empresas logísticas, mucho más allá que el mero transporte de carga. El desarrollo de tales servicios requiere competencia en amplias áreas de experiencia y, materialmente, la construcción de infraestructuras complejas para garantizar el mantenimiento del sistema. Los edificios deben ser funcionales y proporcionar almacenes seguros, los muelles de carga deben ser amplios y estar equipados, y ambos deben colocarse cerca de redes eficientes de transporte por carretera, ferrocarril, mar y aire. Por lo tanto, municipios y regiones se enfrentan hoy, en el contexto de competencia extrema entre empresas logísticas, a un aumento dramático del consumo de suelo urbano. Esto ocurre en varias tipologías de espacios geográficos, como los puertos o los aeropuertos, las afueras de las áreas metropolitanas o las áreas de frontera ubicadas en los principales ejes infraestructurales de transporte.

Así, tomo el ejemplo de la región fronteriza entre el Sur de Suiza (Cantón Ticino) y el Norte de Italia (Lombardía), situada en la ruta del corredor transmarítimo Génova-Rotterdam (ver Figura 1; Torricelli e Garlandini 2018), muy cerca de la región logística de Milán que es, en su conjunto, una de las mayores plataformas de intercambio de bienes en Europa (Dallari e Curi 2010). Como parte de un proyecto encargado por el estado del Cantón Ticino, calculamos el consumo de tierra de las empresas en las zonas industriales: en un asentamiento industrial hay un consumo promedio de suelo de menos de 100 metros cuadrados por empleado (considerando 


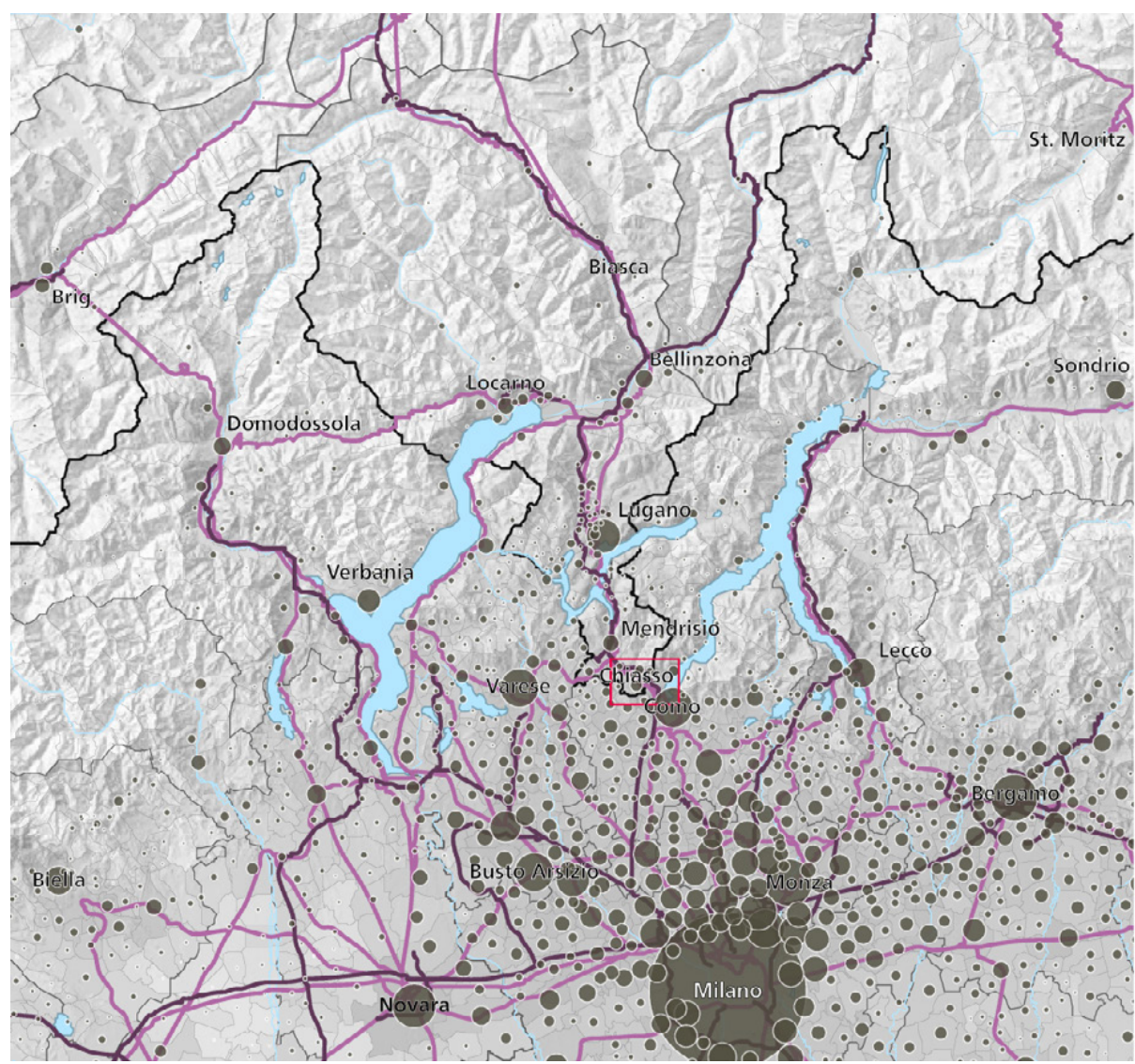

los fondos donde se encuentran las plantas). Por el contrario, en un establecimiento logístico (del mismo tamaño) hay un consumo promedio de suelo de casi tres veces más, entre 250 y 300 metros cuadrados por cada puesto de trabajo. Esto se debe a la automación de muchas tareas (de carga, descarga, etiquetado, monitoreo, etc.) y la necesidad de espacio para el almacenamiento. En el caso propio del almacenamiento, la competencia entre empresas a menudo impide cualquier forma de colaboración entre ellas: como resultado, desde hace algunos años -en ese contexto fronterizo entre Suiza e Italia- estamos asistiendo a la multiplicación de infraestructuras logísticas, a menudo infrautilizadas.

Quizás, sería interesante investigar más el consumo de tierra y comparar diferentes situaciones. Por ejemplo, dónde hay restricciones de planificación (o zonas de logística planeadas) y dónde las áreas industriales son directamente puestas bajo la responsabilidad de los municipios, sin restricciones específicas para actividades que consumen mucho espacio (en función únicamente de planes reguladores locales). Ese tipo de investigaciones serían necesarias hoy, en el contexto de fuerte crecimiento de las actividades logísticas debidas al desarrollo del comercio en línea.

\section{Una relación lábil con el territorio}

Otra pregunta que en el Dossier deja prácticamente sin respuesta (con respeto al desarrollo territorial) concierne a la localización de las empresas de logística y su radicación en los territorios. ¿Cuáles son las lógicas de los asentamientos logísticos en una perspectiva espacial, en particular las relaciones con su entorno (a escala local)? Para su asentamiento, las empresas productoras, y más las de servicio, necesitan externalidades

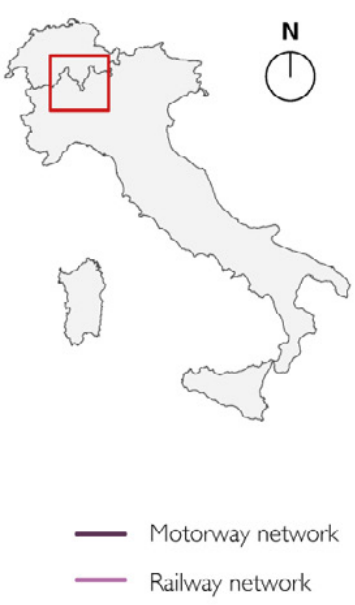

Population in 2014

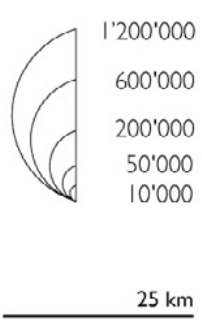

Datx UST, Neurhtel and ISTA Roma Geurnetr. Swisstupo Waberam
Author: OST-TI / AAM-USI.
Figura 1. Distribución de la población entre el sur de Suiza y el norte de Italia. Fuente: Torricelli e Garlandini (2017). 
positivas en un entorno (a escala local o regional) a donde buscan mano de obra, subcontrataciones y servicios especializados. Estas externalidades son un elemento clave de radicación de la empresa en el territorio.

Las empresas de logística actúan, sin embargo, con otra lógica de asentamiento: su desarrollo espacial se construye de acuerdo a las redes en las que están posicionadas, a distintas escalas, sin muchas necesidades de encontrar un entorno local favorable (aparte, la proximidad de los accesos a la red de transporte y la disponibilidad de terrenos construibles). Así, por ejemplo, una empresa logística en Suiza que necesita rápidamente un camión y un chofer para un trasporte entre Italia y Alemania, no lo busca localmente. Esto se encontrará en un nudo de su red (que generalmente es multinacional) según el menor costo. Hoy, en las autopistas que cruzan los Alpes, es fácil encontrar camiones ( $\mathrm{y}$ choferes) de Rumania, Lituania, Polonia, etc. (según las oportunidades y las disponibilidades) para transportar bienes de Italia a Alemania o a Holanda.

Además, la localización de las empresas de logística ocurre, también, por buscar las mejores circunstancias de rentas fiscales. En zonas de frontera, ellas pueden así mudarse rápidamente de una infraestructura a otra en función de distintas oportunidades tributarias y esto tiene un costo para el desarrollo territorial local. De hecho, en estas situaciones, sin respaldo del estado cantonal (en el caso de Suiza) los municipios suburbanos no pueden competir con empresas que actúan a escala global, pues están lógicamente enredados a la escala local.

Por otra parte, los cambios son muy lentos (por ejemplo, en el campo de la planificación local, cualquier medida pide decisiones políticas que toman tiempo) con respeto al dinamismo y a la rapidez de los cambios del mercado. Las consecuencias de este choque de escalas espaciales y temporales -entre las impresas logísticas y los territorios (municipalidades, regiones) donde se encuentran- es algo muy importante e impactante para el desarrollo territorial. Así, en la zona de frontera antes mencionada entre Suiza e Italia, estas empresas - poderosas también en sus actividades de construcción de depósitos especializados- realizan hoy proyectos de construcción de plataformas logísticas perfectamente adaptados a los planes reguladores municipales. Esto, en algunos casos (cómo en las zonas industriales más cerca de la frontera) conduce a una pérdida -parcial o total- del papel regulador de las autoridades locales sobre su jurisdicción territorial y, especialmente, sus áreas industriales. Por supuesto, esto no favorece un desarrollo sostenible. Al contrario, parece fomentar modelos territoriales frágiles, dependientes de rentas fiscales y de las actitudes oportunistas de las empresas multinacionales (a menudo sin relaciones realmente estructuradas con los contextos locales). Si queremos un futuro sostenible, sería crucial hacer comparaciones internacionales en una perspectiva interdisciplinaria, con el fin de proponer medidas adecuadas a cada área involucrada por el desarrollo contemporáneo de la logística. 


\section{Q Bibliografía}

》 DALLARI, Fabrizio y CURI, Sergio (2010) Network Milano: morfologia dei flussi logistici internazionali. Milano: Bruno Mondadori.

»GARCÍA-ORTEGA, María Gabriela, MARTNER-PEYRELONGUE, Carlos, CEDILLO-CAMPOS, Miguel (2017) Análisis espacial de la evolución de los flujos ferroviarios de la industria automotriz mexicana 2011-2013, Revista Transporte y Territorio 17, pp. 100-116.

» GONZÁLEZ-FELIU, Jesús y CEDILLO-CAMPOS, Miguel (2017) Presentación Dossier. Logística y desarrollo territorial, Revista Transporte y Territorio 17, pp. 1-9.

» MUÑUZURI, Jesús, GROSSO, Rafael, ESCUDERO, Alejandro y CORTÉS, Pablo (2017) Distribución de mercancías y desarrollo urbano sostenible, Revista Transporte y Territorio 17, pp. 34-58.

» PACHÓN RINCÓN, Mateo, SUÁREZ MORENO, Juan y ORTIZ TRIVIÑO, Jorge (2017) Análisis de un esquema de coordinación para los procesos de descargue en grandes almacenes, basado en modelos por eventos discretos, Revista Transporte y Territorio 17, pp. 59-76.

"PALACIOS-ARGÜELLO, Laura, MORGANTI, Eleonora y GONZALEZ-FELIU, Jesús (2017) Food hub: Una alternativa para alimentar las ciudades de manera sostenible, Revista Transporte y Territorio 17, pp. 10-33.

» RAIMBAULT, Nicolas, DOUET, Marie et FREMONT, Antoine (2013). Les implantations logistiques entre réseaux et territoires. L'Espace géographique, 1(42), pp. 32-43.

" ROSADO DE SOUSA, María, LÁZARO DA SILVA FILHO, José, HERBERTHY MARTINS FERREIRA, Johnny (2017) FAIR TRADE - uma certificação que contribui com o desenvolvimento sustentável: o caso da CASA APIS do Brasil, Revista Transporte y Territorio 17, pp. 77-99.

» TORRICELLI, Gian Paolo e GARLANDINI, Simone (2017) La frontiera e le mappe. Evidenze demografiche e socioeconomiche tra Svizzera e Italia, 2010-14. Quaderni OST-TI, I-2017, Dipartimento del territorio, Bellinzona e Università della Svizzera italiana, Mendrisio http://www.arc.usi.ch/it/ricerca-e-istituti/istituti/ ost/quaderni).

» TORRICELLI, Gian Paolo e GARLANDINI, Simone (2018, de próxima publicación en línea) Nuove geografie della logistica nel Cantone Ticino. Quaderni OSTTI, II-2017, Dipartimento del territorio, Bellinzona e Università della Svizzera italiana, Mendrisio http://www.arc.usi.ch/it/ricerca-e-istituti/istituti/ost/ quaderni.

\section{Gian Paolo Torricelli}

Geógrafo. Doctor. Director del Observatorio del desarrollo territorial (Osservatorio dello sviluppo territoriale / OST: www.arc.usi.ch/ost) y titular de los cursos de geografía urbana y de desarrollo territorial (Master) en la Academia de Arquitectura de la Università della Svizzera italiana (USI) en Mendrisio (Suiza). Fue profesor visitante en las universidades de Ginebra, Milán, Grenoble y Buenos Aires. 